\title{
Ectopic breast tissue in the vulva masquerading as an abscess
}

Singapore Med J 2020; 61(5): 281-282 https://doi.org/10.11622/smedj.2018121

Dear Sir,

Ectopic breast tissue (EBT) along the embryonic milk lines is reported to be more common in Asian women. Hormonal changes associated with puberty, pregnancy or lactation can result in functional activity within the dormant glandular tissue, leading to symptoms. We describe the case of a 34-year-old Chinese woman who presented with a suspected vulvar abscess. Based on our literature search, this is the first case of vulvar EBT presenting as an abscess.

The patient presented with a three-week history of left vulvar swelling. Although she had noted the vulvar swelling for the past two years, there had been a substantial increase in its size, accompanied by pain and discharge, over the past three weeks. She had undergone a lower-segment Caesarean section one month prior to this presentation and was currently breastfeeding a healthy baby. On examination, the patient was afebrile, and an erythematous, tender, fluctuant left labia majora swelling $(5 \mathrm{~cm} \times 4 \mathrm{~cm})$ with purulent discharge was observed. She was diagnosed with a vulvar abscess and underwent incision and drainage under local anaesthesia. Intraoperatively, a cystic lesion of the left labia that contained whitish purulent material was evident. The entire cyst wall was excised and sent for histology. The histology report showed EBT with lactational changes and acute-on-chronic inflammation (Fig. 1). Postoperatively, she recovered well and was managed with dressing in the outpatient setting. Three months after surgery, she was seen in the outpatient clinic. On physical examination, the wound had healed with no ectopic tissue identified anywhere along the milk lines, and she was discharged from follow-up.

Early in the development of the embryo, two lines develop along its lateral aspects stretching from the axilla to the groin. These lines develop into mammary ridges, which will be the future site of the breasts. Most of these ridges regress under normal circumstances except for imminent breast tissue. EBT is a congenital anomaly in which abnormal additional breast tissue is present along the mammary/milk lines. This occurs more frequently in females and is more common among Asians compared to Caucasians. According to one study, the incidence of EBT can be as high as $5 \%$ in Japanese females. ${ }^{(1)}$ There is some evidence that this condition may be inherited. (2) EBT remains asymptomatic until the patient is under the hormonal influences of puberty, pregnancy or lactation. ${ }^{(3)}$ Copeland and Geschickter classified EBT with an accessory nipple or areolar formation with or without glandular tissue as supernumerary breast. EBT without a nipple or areolar complex is classified as aberrant breast tissue. ${ }^{(4)}$ The most common site is the axilla, which accounts for $60 \%-70 \%$ of the cases. Vulval EBT is extremely rare, with only 12 cases ${ }^{(5)}$ reported thus far, based on a literature search.

EBT manifests all the different features of normal breast tissue such as growth during puberty, pregnancy and lactation, as well as pathologies such as benign and malignant tumours. ${ }^{(6)}$ EBT may become symptomatic during periods of hormonal stimulation such as during puberty (menarche), pregnancy and lactation. The symptoms may manifest as swelling, pain, discomfort or lacteal secretions. These changes may be cyclical, coinciding with menstruation. Rarely, the EBT may undergo malignant change. Ectopic breast cancer is very rare, constituting $0.3 \%-0.6 \%$ of all breast cancers. ${ }^{(7)}$

Surgical excision is recommended for symptomatic EBT.(2) This would relieve symptoms and allow for histological confirmation of the diagnosis. Liposuction has been described as an option in patients with axillary EBT in whom cosmesis is a priority. ${ }^{(1)}$ In asymptomatic patients with vulvar EBT, there is no clear consensus on management. Some authors recommend surgical excision owing to the risk of malignant transformation; however, this is debatable given that the risk of transformation is generally low. ${ }^{(5)}$
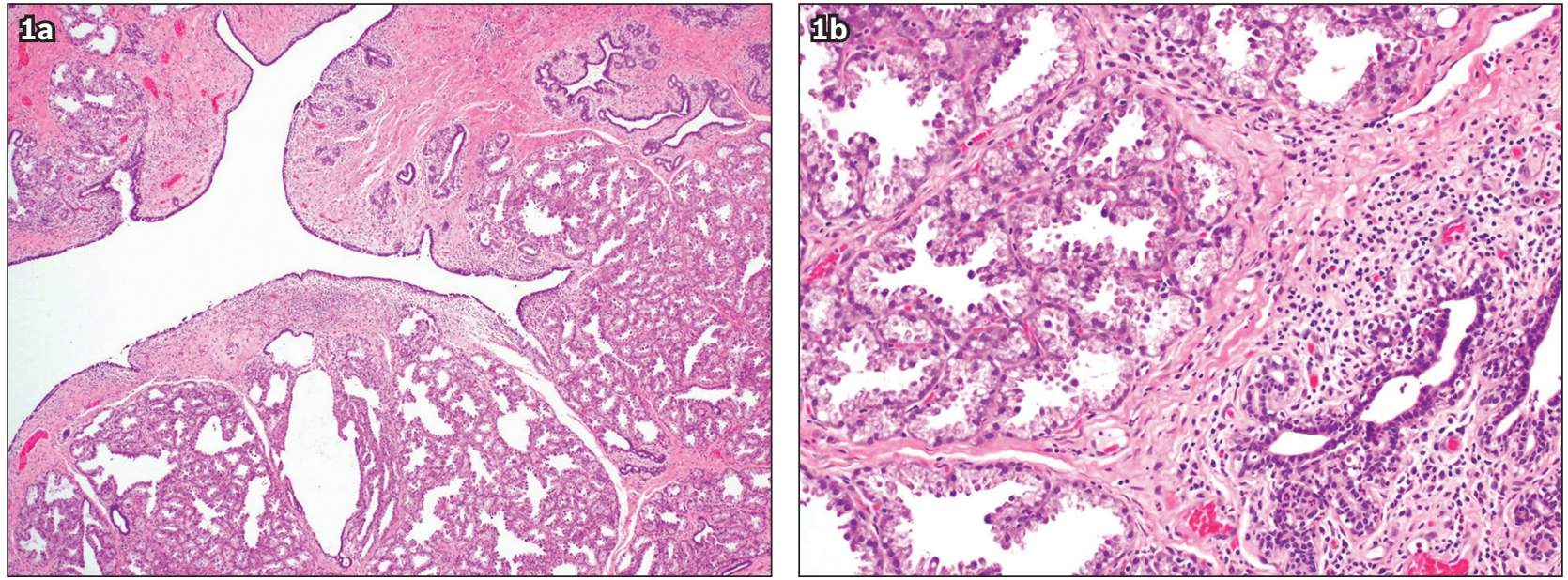

Fig. 1 Photomicrograph of the cystic lesion shows (a) cystically dilated spaces that are lined by bi-layered ductal epithelium, accompanied by breast ducts and lobules of breast acini in the adjacent area (Haematoxylin \& eosin, $\times 40$ ) and (b) acute-on-chronic inflammation and lactational changes of breast acini (Haematoxylin \& eosin, $\times 200)$. 
In our case, the patient likely developed symptoms coinciding with the start of lactation. The preoperative clinical diagnosis was that of a vulvar abscess, and surgical drainage was recommended under general anaesthesia. As the patient wanted to continue breastfeeding in the immediate postoperative period, she insisted on surgery being performed under local anaesthesia. During surgery, whitish purulent material was drained. Due to the unusual nature of the abscess wall, which was akin to a cyst cavity, a decision was made to subject the tissue to histology. In the 11 cases of vulvar EBT reviewed by Godoy-Gijón et al in the literature, all except one were treated with excision. The majority of the patients were aged below 30 years, and all except two were of childbearing age. ${ }^{(5)}$

The diagnosis of EBT is usually made postoperatively due to the rare nature of this condition. Differentials of vulvar mass include abscess, vulvar carcinoma, Bartholin gland disorder and epidermal cyst. Cyclical changes in the mass during menstruation or the onset of symptoms during pregnancy support the diagnosis of EBT.

In conclusion, in patients with a mass along the milk line, especially with coincident hormonal changes, an index of suspicion is warranted for underlying EBT. Surgical excision is recommended in symptomatic patients.

Yours sincerely,

Saleem $\underline{\text { Ahmed }}^{1}$, Cristine SL $\underline{\text { Ding }}^{2}$, Vishal G $\underline{\text { Shelat }}^{1}$

${ }^{1}$ Department of General Surgery, ${ }^{2}$ Department of Pathology, Tan Tock Seng Hospital, Singapore. medicsaleem@gmail.com

\section{References}

1. Patel PP, Ibrahim AM, Zhang J, et al. Accessory breast tissue. Eplasty 2012; 12:ic5.

2. Sahu S, Husain M, Sachan P. Bilateral accessory breast. Internet J Surg 2007; 17:4-7.

3. Howard BA, Gusterson BA. Human breast development. J Mammary Gland Biol Neoplasia 2000; 5:119-37.

4. Copeland MM, Geschickter CF. Diagnosis and treatment of premalignant lesions of the breast. Surg Clin North Am 1950; 30:1717-41.

5. Godoy-Gijón E, Yuste-Chaves M, Santos-Briz A, Esteban-Velasco C, de Unamuno-Pérez P. [Accessory breast on the vulva]. Actas Dermosifiliogr 2012; 103:229-32. Spanish.

6. Mak CT, Veras E, Loveless MB. Supernumerary nipple presenting as a vulvar mass in an adolescent: case report and literature review. J Pediatr Adolesc Gynecol 2009; 22:e41-4.

7. Kitamura K, Kuwano H, Kiyomatsu K, et al. Mastopathy of the accessory breast in the bilateral axillary regions occurring concurrently with advanced breast cancer. Breast Cancer Res Treat 1995; 35:221-4. 